\title{
Using a screening tool to improve timely referral of patients from acute oncology-haematology to palliative care services
}

\author{
Akhtari Begum
}

\begin{abstract}
This project was done at specialist cancer hospital in Qatar. At a haematology-oncology inpatient department most patients were not getting access to palliative care unless they were at the very end stages of life. Data collected from 2008-2011 showed significant numbers of patients were dying within one month of their transfer to palliative care. There was no standard measure to identify the prospective palliative care patients. A multidisciplinary team developed a Palliative care referral screening tool based on the National Cancer Care Network guideline. Retrospective medical record review done from January to April 2012 showed a mean of $68 \%$ of patients who scored more than five were not consulted, $32 \%$ of patients who scored more than seven were not transferred to palliative care and seven percent died without any referral. The team used various kinds of quality planning, analysis and improvement tools in the form of process mapping, value analysis, Fish Bone diagrams, stakeholders' analysis and communication, physician survey, "Pareto's principal" (80 / 20 rule, the law of vital few) and other data collection tools.
\end{abstract}

The palliative care referral process was standardised by preparing and implementing an objective scoring tool based on international best practice. It changed the referral culture and helped manage the psychological barriers of patients, families and caregivers. Extensive orientation and education of all key stakeholders was implemented. Monthly auditing of patient records was carried out. The aim has been achieved, exceeded and sustained, and we reduced the percentage of patients who scored more than five without palliative consultation from a mean of $68 \%$ to $16 \%$ and those who scored more than seven without palliative care transfer from a mean of thirty two percent to three percent, after four months of the project's implementation.

Standardising the referral process and creating an objective referral tool is needed to facilitate safe, collaborative, continuous and patient centered care. Timely referral of cancer patients to palliative care minimises patient and caregiver distress, ensures better quality of life, and provides an appropriate measure for end of life care.

\section{Problem}

Most of the patients with cancer were not getting access to Palliative care services at an appropriate time in their disease trajectory. There was no standard measure to identify the prospective palliative care patients. Consultation of patients and their families about palliative care was not done except for terminally ill patients, just before transfer to palliative care services, which compromised quality of life.This Improvement project was done at specialist cancer hospital in Qatar.

\section{Background}

This project was done under the guidance of the Clinical Care Improvement Training program ( CCITP ). A multidisciplinary team was formed and a literature review was carried out to prepare a business case to clearly define our project. A data collection plan was prepared for our project which started in February 2012.

While being treated for cancer, challenging situations, like insurmountable physical distress, inadequate coping patterns and unanswered spiritual issues, leads to a debilitating Quality of life. The Palliative Care team approach addresses all these issues and also sees the patient to go through the protocols of Palliative care management as well as Oncology treatment plan. Further, this facilitates a smooth transition from the hospital to home and hospice care. (1)

In a randomised controlled study conducted in Lebanon, 322 patients newly diagnosed with advanced cancer from November 2003 through May 2008, who received palliative care interventions, had higher scores of Quality Of Life ( QOL) and mood, compared to patients who received only oncology care. (2) One study found that palliative care consultations were associated with an average saving of $\$ 1700$ per admission for patients who were discharged, and $\$ 4900$, on average, for every patient who died in the hospital.(3) Patients with advanced lung cancer who received integrated palliative care early on during treatment had a better QOL and survived for two months longer (11.6 months versus 8.9 months) compared to patients receiving standard care (chemotherapy) according to a study published in the August 19, 2010 issue of New England Journal of Medicine. (4)

\section{Baseline Measurement}

The multidisciplinary team developed a palliative care referral screening tool based on the National Cancer Care Network guideline (5). A score of more than five meant the patient should have a Palliative care consultation and seven meant palliative care 
transfer. Retrospective medical record reviews were done from January 2012 to April 2012 and showed a mean of $68 \%$ patients who scored more than five were not consulted and $32 \%$ patient who scored more than seven were not transferred to palliative care. Seven percent of them died without any consultation or transfer to palliative care.

See supplementary file: ds1620.ppt - "Attachment 1 showing pie chart with shortfall ,palliative care referral tool and bar graph showing problem"

\section{Design}

A process flow map was created and waste analysis carried out. In the process there were 6 wastes, 5 enablers, 4 values added and 2 hands off activities. Value add is a Lean term; a value added step is a step that the patient is willing to pay, for example diagnosis of cancer, starting first line treatment and transfer to palliative care set up.

Enabler are step that are considered necessary by organization for the quality and safety reason but the patient see it as waste for example multidisciplinary team assessment, staging, plan of care, policy, screening tool, consent form etc.

Waste is the step in process which is considered as non value adding in the process map for example unnecessary exposure of patients to radiotherapy and chemotherapy with second line and third line therapy.

Hands off is handing over to another department ( for example from acute care to palliative care).

The team brainstormed to prepare a Cause and Effect diagram for the delayed referral and consultation of cancer patients to palliative care.

The two most important causes identified were that there was no availability of Palliative care referral criteria and patients and family were resisting referral to palliative care. A survey was done among physicians to buy in physician support and identify the causes of non referrals. Pareto's principle was used to analyse the survey results and to identify the cumulative percentage of $20 \%$ of barriers leading to $80 \%$ of the problem. The most important cause identified from the survey was that doctors not being aware of how to assess and manage palliative care needs. Among their common comments the most stated were 'It's too early' or 'Let's give it some more time' etc. Feedback from the physicians during the survey were supportive and positive about our palliative care referral screening tool.

Process redesign was carried out by implementing the palliative care referral screening tool (with referral criteria) to be filled in at every admission for all patients with cancer.
A great amount of time was dedicated in educating physicians, residents, nurses, medical record staff and admitting clerks on this subject and the suggested improvement. Baseline data were presented to physicians during morning reports. Patient and family education materials/brochures were prepared in collaboration with the palliative care team to overcome barriers in palliative care referral.

After implementing the new referral process, there were extensive consultations and transfers of patients to palliative care. There was a sudden increase in the demands of services and a decrease in the supply (beds, staff and other resources). Through a collaborative effort among Haematology, Oncology and Palliative care teams, this was successfully managed. There were a series of meetings, discussions and data presentations and it was mutually agreed to gradually transfer patients to palliative care. Each team would transfer one patient per week until additional resources were allocated and arranged to handle more patients.

By implementing early screening of patients for palliative care consultation and transfer it allowed us to provide better quality of life to the cancer patients and reduce waste. Our referral tool allowed us to reduce waste of resources and and minimise unnecessary exposure of patients to radiation and chemotherapy, while improving pain management and end of life care. Standardisation of process allowed us to identify patients' needs for palliative services at admission.

\section{Results}

Prospective and retrospective data were collected through 30 medical record reviews done monthly to identify the percentage of patients who scored more than, five or seven and who were not consulted or transferred to palliative care.

We reduced the percentage of patients with scores of more than five without palliative consultation from a mean of $68 \%$ to $16 \%$ and with scores of more than seven without care in palliative setting from a mean of thirty two percent to three percent after four months of project implementation. By standardising the palliative care referral process, we achieved our aim by reducing nonreferral/consultation.

This had the following impact on our healthcare system:

Timely patient care leads to:

- Assuring continuity and access to palliative care and consultation on a timely basis.

- Increased patient satisfaction

- Improved the quality of life

- Better survival

- Standarded care processes

\section{Strategy}


- Minimising patient and caregiver distress

- Increased awareness and decreased resistance

- Improved communication among teams.

- Helped us with the job planning inside our facility and guided us to better use resources.

Patient Safety:

- Reduce exposure to chemotherapy

- Reduce exposure to radiation

Efficiency and Reduce waste:

- Reduced unit cost

- Reduced length of stay

- Reduced admission and readmission rates

See supplementary file: ds1622.ppt - "Old and new process map, Fish bone diagram, survey results ( Pie and Pareto chart) and Run chart with improvement results"

\section{Lessons and Limitations}

Involvement of all the key stakeholders reduced resistance to change. Continuous reminders, presentations and open access to data was required to sustain this project. Increase in palliative care consultation and transfer resulted in the increase in the demands of staff and other resources. Capacity and demand analysis was essential to smoothly implement and sustain this project.

\section{Conclusion}

The data shows a clear increase in the number of consultations and transfers to palliative care earlier using the scoring tool and the newly implemented process. This was not usual practice before our project, where acute Oncology - Haematology teams missed such consultations, depriving their patients from the privilege of specialised palliative services and the benefits mentioned above. Standardising the referral process and creating objective referral tools are needed to facilitate the transfer of care from one level to another, in a patient centered and collaborative way. The process improves management of psychological, social, and spiritual issues associated with living with cancer. Timely referral to palliative care minimises patient and caregiver distress and ensures better quality of life, patient safety and appropriate measures at the end of life.
2. Bakitas M, Lyons KD, Hegel MT, Balan S, Brokaw FC, Seville $\mathrm{J}$, et al. Effects of palliative intervention on clinical outcomes in patients with advanced cancer. JAMA 2009;302:741-9

3. Morrison RS, Penrod JD, Cassel JB, et al. Cost savings associated with US hospital palliative care consultation programs. Arch Intern Med. 2008;168:1783-1790

4. Temel JS, Greer JA, Muzikansky A, Gallagher ER, Admane $S$, Jackson VA, et al. Early palliative care for patients with metastatic non- smallcell lung cancer. N Engl J Med 2010;363:733-42

5. Palliative Care in the Outpatient Oncology Setting: Evaluation of a Practical Set of Referral CriteriaBy Paul A. Glare, MBBS, MM, FRACP, FACP, Deborah Semple, RN, Stacy M. Stabler, MD, MPH, and Leonard B. Saltz, MDMemorial Sloan-Kettering Cancer Center, New York, NY

\section{Declaration of interests}

Nothing to declare.

\section{Acknowledgements}

Team members : Dr. Mufid M El Mistri, Dr. Kamal Aboudi, Ms. Amra Alkhalil, Dr. Reham H. Negmeldin, Dr. Hanadi Al Ayoubi, Dr .Azza Hassan, Dr. Saad Eziada. 